\title{
A study of the stored energy in titanium under deformation and failure using infrared data
}

\author{
A.Yu. Fedorova, M.V. Bannikov, O.A. Plekhov \\ Institute of Continuous Media Mechanics of the Ural Branch of the Russian Academy of Sciences, 614013, Ac. Koroleva Street, \\ 1, Perm, Russia \\ poa@icmm.ru
}

\begin{abstract}
The work is devoted to the experimental study of heat dissipation caused by plastic deformation and failure processes taking place in a titanium alloy Ti-4.2Al-1.6Mn. To investigate the spatial and time evolution of temperature, a set of experiments has been carried out on plane titanium smooth specimens and specimens with pre-grown centered fatigue cracks. The original mathematical algorithm for experimental data processing has been applied to obtain the rate of heat dissipation generated by plastic deformation and stored energy. It is shown that the stored energy is accumulated in titanium specimens undergoing fatigue tests, and at the time of damage to fracture transition it is equal to zero.
\end{abstract}

KEYWORDS. Heat dissipation; Infrared thermography; Stored energy.

\section{INTRODUCTION}

I $\mathrm{t}$ is well known that real metals have a complex structure, which is a hierarchy of different scale levels $[1,2]$. Under deformation, the structural evolution is observed at all scale levels and leads to irreversible deformation and failure that is accompanied by energy accumulation and dissipation. Investigation of thermodynamics of deformation and failure is a key issue in solid mechanics. These studies allow one to develop a universal material failure criterion for estimating different stress-strain states (including multi-axial ones) and loading histories.

The experimental and theoretical study of the energy balance during deformation is based on an extensive bibliography. The importance of this problem was originally shown by J. H. Lambert in 1779 in his work concerning the energy similarity of mechanical and thermal failure processes of solids. The essence of this similarity is that the mechanical failure of metals can occur when the relative deformation in at least one direction reaches a value equal to the linear thermal expansion at the melting point. A substantial contribution to the development of these ideas was made by V.S. Ivanova, who proposed the structure-energy theory of metal fracture [3]. A detailed analysis of thermodynamic effects produced by cyclic deformation and failure in metals was carried out by V.T. Troshchenko and V.V. Fedorov. A review of strength energy criteria was given in $[4,5]$.

To analyze the thermodynamic characteristics of deformation processes in solids, it is necessary to take into account the fact that the plastic deformation work is converted into two parts: heat energy caused by the movement and annihilation of defects at various structural levels, and hidden (stored) energy of plastic deformation accumulated in the elastic fields of defects. The energy is dissipated in the surrounding environment by conduction, convection and radiation and also stored in the material as thermal energy that increases due to the self-heating effect.

The main difficulty encountered in the analysis of deformation and failure processes is a choice of thermodynamic parameters required to determine the exhaustion degree of material deformability. In work [5] published in 1979, it was experimentally shown that an endurance limit can be determined by using almost any thermodynamic characteristics of the process: inelastic deformation per one load cycle, irreversibly consumed energy per one load cycle, self-heating 
temperature, energy dissipation rate, and energy storage rate. For a more rapid determination of the endurance limits of metals, the author proposed two types of tests: a slowly increasing load (stress) amplitude test and a block amplitude test (used more often today). In the beginning of 1970th, V. Fedorov developed an original experimental setup that permitted him to control the heat dissipation and to measure the temperature of the specimen under cyclic loading.

The analysis of the experimental studies carried out in USSR in 1970-1980 has led us to the conclusion that the obtained parameters are indirect and thus cannot be used as a basis for the development of the universal material failure criteria.

For example, it is experimentally shown [5] that the thermal energy dissipated during the cyclic deformation of specimens made from 40X, 2X10, 25, 45 steels (Russian marking) may have very different values [5]. Conversely, the value of the stored energy during deformation is independent of loading conditions and correlates, at the time of failure, with the value of enthalpy of the material in a liquid state at melting temperature.

With the advent of infrared cameras it became possible to significantly simplify the schemes of experiments and to accelerate the determination of fatigue limits of materials [6-8]. Infrared thermography allows one to measure, in real time, the temperature of materials under deformation and to calculate the thermal energy of specimens and the rate of heat dissipation.

Investigation of the energy accumulation process in metals at different loading conditions was carried out over the entire 20th century. The review of experimental works devoted to the methods of studying the stored energy in the material under deformation and the peculiarities of this process for different materials and load conditions is available in [9]. Currently, it has been convincingly shown that the ratio of the energy storage rate to the plastic work rate can reach a value of 0.3-0.4 at the initial stages of plastic deformation process [10,11].

The aforementioned experimental results indicate the importance of further development of methods for investigation of the thermodynamic parameters of the deformation process. At present, infrared thermography is one of the most promising techniques for thermodynamic measurements; it requires no changes in the standard schemes of mechanical testing.

One of the main problems facing scientists today is the development and realization of mathematical methods for experimental data processing, including noise filtering for temperature measurements based on infrared radiation data, elimination of external effects during the experiments (reflection of infrared waves from the metal specimen surface, relative motion of a specimen, etc.), and calculation of heat losses in the course of the experiment. The major goal of such experimental data processing is to provide a solution to the inverse problem and to evaluate the heat source evolution caused by the structure evolution in the material. A comparison of these data with the results of mechanical tests will enable one to determine the value of stored energy and to propose a local material failure criterion. This criterion can be applied for calculation of the stored energy value and determination of the moment at which this parameter is equal to zero, that is, the time of local material failure. In the present paper, the proposed criterion is used in the analysis of stress field and energy dissipation at the fatigue crack tip.

\section{MATERIALS AND CONDITIONS OF EXPERIMENT}

7 he experimental study of temperature evolution at the fatigue crack tip was carried out on the two types of the plane specimens of titanium alloy. The chemical composition of the material is presented in Tab. 1. Experiments were made on smooth specimens and specimens weakened by holes to initiate fatigue cracks in the center of the specimen. The specimens were manufactured from a titanium sheet $3 \mathrm{~mm}$ thick. The mechanical properties of the materials were determined based on the original experiment. Mechanical tests were carried out using a $100 \mathrm{kN}$ servohydraulic machine Bi-00-100. The mechanical properties and fatigue loading conditions are presented in Tab. 2. The geometry of specimens is shown in Fig. 1.

\begin{tabular}{ccccccccc}
\hline $\mathrm{Al}$ & $\mathrm{Zr}$ & $\mathrm{Si}$ & $\mathrm{Fe}$ & $\mathrm{O}$ & $\mathrm{H}$ & $\mathrm{N}$ & $\mathrm{C}$ & $\mathrm{Mn}$ \\
3.68 & 0.3 & 0.12 & 0.3 & 0.15 & 0.012 & 0.05 & 0.1 & 1.16 \\
\hline
\end{tabular}

Table 1: The chemical composition of titanium alloy $(\%)$.

\begin{tabular}{cccccccc}
\hline $\begin{array}{c}\text { Modulus of } \\
\text { elasticity }\end{array}$ & Yield stress & $\begin{array}{c}\text { Ultimate } \\
\text { stress }\end{array}$ & $\begin{array}{c}\text { Fatigue } \\
\text { limit }\end{array}$ & $\begin{array}{c}\text { Fracture } \\
\text { toughness }\end{array}$ & $\mathrm{P}_{\min }$ & $\mathrm{P}_{\max }$ & $\begin{array}{c}\text { Stress } \\
\text { ratio }\end{array}$ \\
$64 \mathrm{GPa}$ & $800 \mathrm{MPa}$ & $900 \mathrm{MPa}$ & $460 \mathrm{MPa}$ & $75.6 \mathrm{MPa} \sqrt{m}_{\mathrm{m}}$ & $-18.7 \mathrm{MPa}$ & $367 \mathrm{MPa}$ & -0.051 \\
\hline
\end{tabular}

Table 2: The mechanical properties of Ti-4.2Al-1.6Mn and fatigue loading conditions. 
The test conditions comply with the conditions of the experiment described in detail in [12]. Investigation of the heat source evolution was carried out on the smooth specimens during a quasi-static tensile test. Crack propagation was studied at loading frequency of $5 \mathrm{~Hz}$ and $10 \mathrm{~Hz}$. The temperature evolution was recorded by an infrared camera FLIR SC 5000. The spectral range of the camera is $3-5 \mathrm{~mm}$. The maximum frame size is $320 \times 256$ pixels; the spatial resolution is $10-$

${ }^{4}$ meters. The temperature sensitivity is from $25 \mathrm{mK}$ to $300 \mathrm{~K}$.

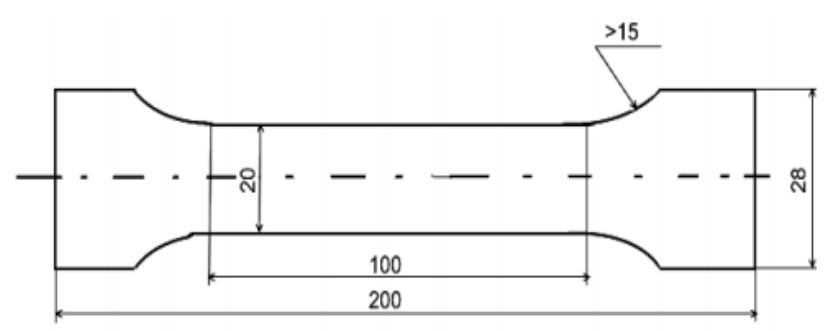

(a)

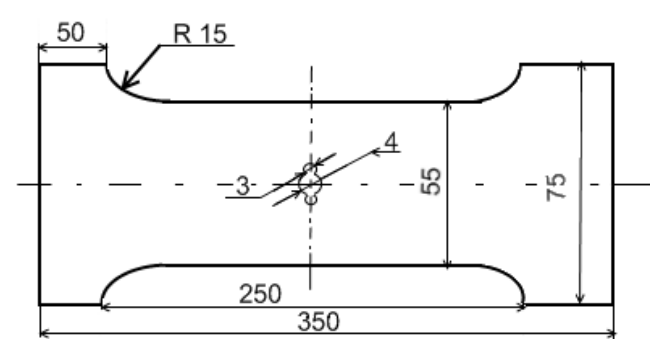

(b)

Figure 1: Geometry of specimen ( $\mathrm{a}$ - smooth specimen, $\mathrm{b}$ - specimen with a hole). All sizes are in millimeters.

\section{EXPERIMENTAL DATA PROCESSING AND STORED ENERGY DETERMINATION}

\section{Post experimental data processing}

A the beginning of the data processing procedure, the first frame was subtracted from the film to eliminate the 1 influence of infrared camera lens radiation on the temperature field. To increase data accuracy and to eliminate the influence of random temperature fluctuations, the spatially fixed temperature signal of the smooth specimen was processed using the two-dimensional discrete Fourier transform with a standard Gaussian kernel [13]. The result of such data processing used to calculate the heat source field is illustrated in Fig. 2.

The relative motion of the specimen with cracks and the infrared camera lens observed in cyclic tests causes the problem of motion compensation, which should be overcome to obtain the correct temperature data at the given point on the specimen surface. To compensate this relative motion, the algorithm described in detail in [13] was used. The main idea of the algorithm consists in finding a marker zone on the examined surface and searching for this area on the surface in each time step. Further, the displacement of every point on the surface is calculated for each time step. The data processing yielded the temperature increment field (Fig. 3), which was used to determine the heat source field.

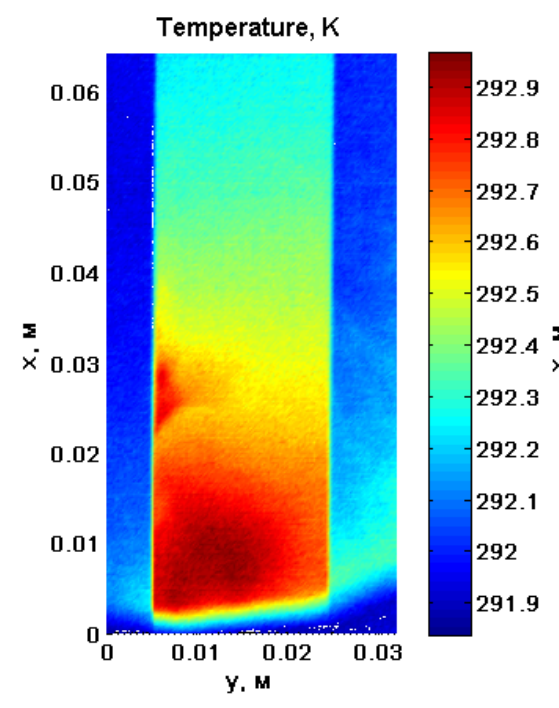

(a)

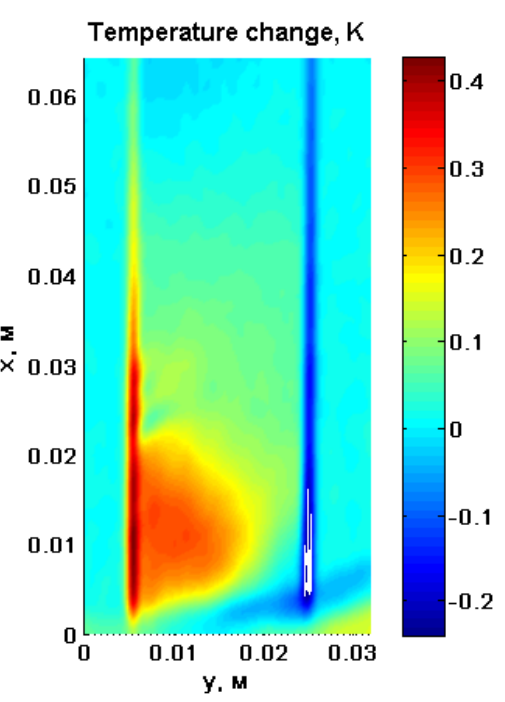

(b)

Figure 2: Infrared image of the smooth specimen before data processing (a) and the obtained temperature change field (b). 


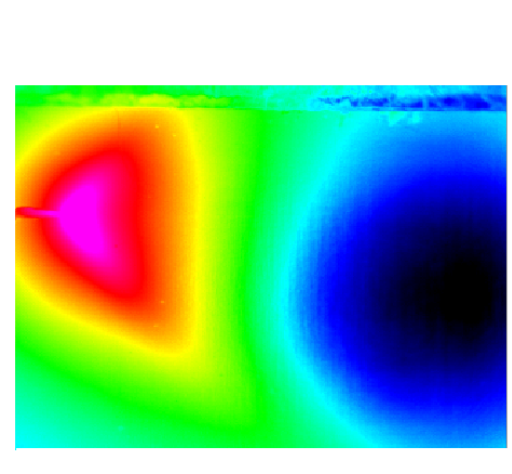

(a)

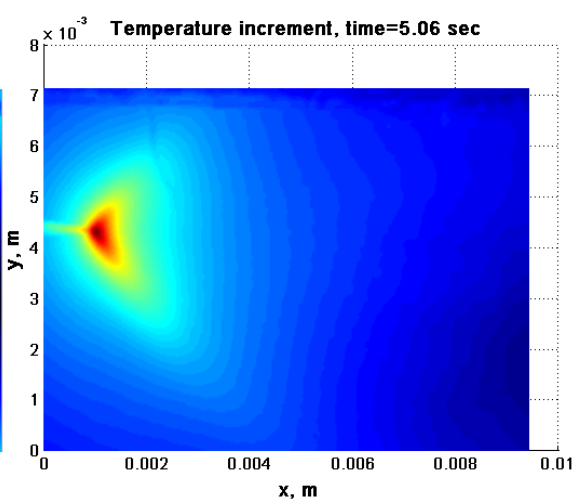

(b)

Figure 3: Infrared image of the specimen with crack before data processing (a) and the obtained temperature increment field (b).

\section{Calculation of stored energy under quasistatic loading}

To calculate the specific power of heat source, we have used the finite difference scheme of equation (1) for heat source evolution

$$
s=\rho c\left(\dot{T}+\frac{T}{\tau}\right)-k \Delta T
$$

where $T$ is the temperature, $\varrho$ is the density $\left(4550 \mathrm{~kg} / \mathrm{m}^{3}\right), c$ is the heat capacity $(600 \mathrm{~J} /(\mathrm{kg} \cdot \mathrm{K}))$, $k$ is the heat conductivity $(6.5 \mathrm{~W} /(\mathrm{m} \cdot \mathrm{K})), s$ is the unknown specific power of heat source $\left(\mathrm{W} / \mathrm{m}^{3}\right)$, and $\tau$ is the constant corresponding to the heat loss due to heat exchange with the surroundings $\left(103 \mathrm{~J} /\left(\mathrm{m}^{3} \cdot \mathrm{K}\right)\right)$.

The power of heat source at time close to fracture is shown in Fig. 4. The plastic zone is localized on the surface of the smooth specimen (Fig. 4a). Fig. 4b presents the last moments before fracture of the specimen with crack; one can observe strong plastic deformation at the crack tip, and the plastic zone has the form of a "butterfly".

Here we also use the assumption $[14,15]$ that some of the irreversible plastic work contributes to heat generation, while the rest is stored as the energy of crystal defects accompanying plastic deformation, traditionally known as the stored energy of cold work.

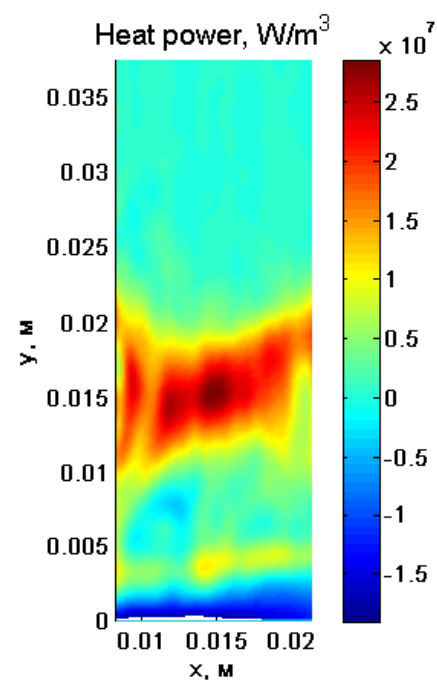

(a)

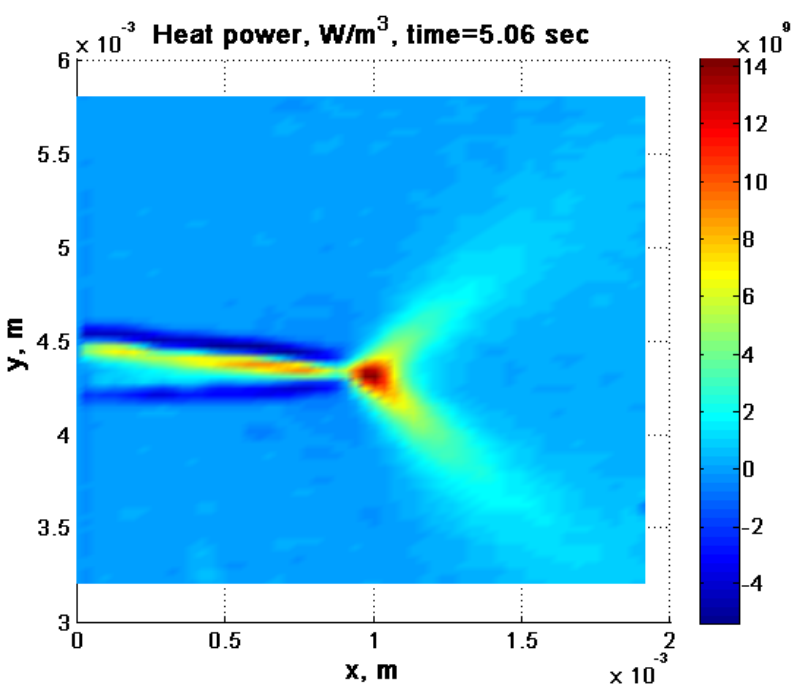

(b)

Figure 4: Experimental data for the heat power field near the crack tip at the beginning of unstable crack propagation.

For plane specimens without crack the plastic work under quasi-static tensile loading is calculated using the experimental data obtained for the applied force $F(t)$ and the deformation velocity $V$ : 


$$
W_{p}^{q u a s i}(t)=F(t) V
$$

The power of heat source is integrated over the plastic localization zone, and the heat dissipation energy is defined by the equation

$$
Q^{q u a s i}(t)=\int_{x 1}^{x 2} \int_{y 1}^{y_{1}} s(x, y, t) d x d y
$$

where $x_{1}, x_{2}, y_{1}, y_{2}$ are the rectangular coordinates of the plastic deformation zone.

The stored energy is determined as a difference between the plastic work and the heat dissipation energy. The results of calculation are presented in Fig. 5.

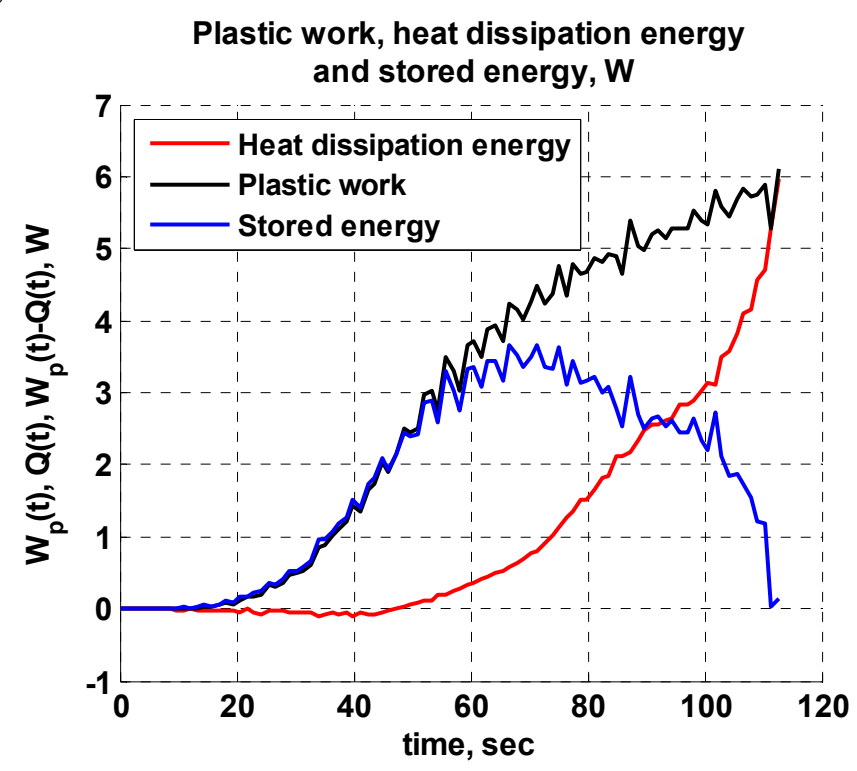

Figure 5: Time dependence of plastic work, heat dissipation energy and stored energy calculated for the smooth specimen.

By analyzing the data presented in Fig. 5, we can conclude that the stored energy rate tends to zero at the time of failure. This supports the results [5] indicating that the stored energy is a thermodynamic parameter, which can adequately describe the damage evolution in metals under deformation.

\section{Calculation of stored energy at fatigue crack tip}

To define the plastic work at the fatigue crack tip, we have used the solution for stress distribution at the crack tip obtained by Hutchinson, Rice and Rosengren (HRR-solution). The specific plastic work in the direction of crack propagation can be written as [16]:

$$
w_{p}(r, \theta, t)=\int_{0}^{\varepsilon_{p}} \sigma d \varepsilon=\frac{n}{n+1} \frac{J(t) \sigma_{e}^{n+1}(\theta, n)}{I_{n} r}
$$

where $n$ is the hardening coefficient (in our case, $n=4), I_{n}$ is the function of the hardening coefficient, $r$ and $\theta$ are the polar coordinates of the point near the crack tip $(x=r \cos \theta, y=r \sin \theta), \sigma_{e}$ is the tabulation function, and $J(t)$ is the energy J-integral that is the function of applied cycling loading and crack length. The time dependence of the J-integral is plotted in Fig. 6. The stored energy is obtained as a difference between the accumulated plastic work and the accumulated heat dissipation energy calculated in the area near the crack tip at all time moments of the experiment. Thus, we have

$$
\begin{aligned}
& W_{p}(x, y, t)=\int_{0}^{t_{i}} w_{p}(x, y, t) d t \\
& Q(x, y, t)=\int_{0}^{t_{i}} s(x, y, t) d t
\end{aligned}
$$




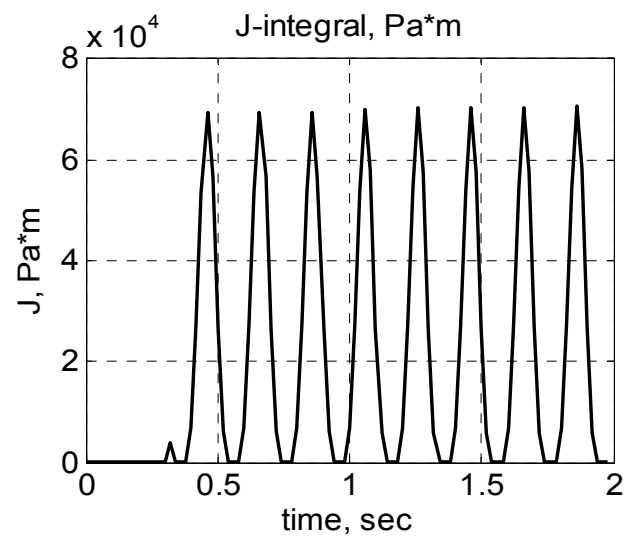

Figure 6: Time dependence of the energy J-integral under cycling loading.

The time dependence of accumulated plastic work, dissipative energy and stored energy calculated for the moving fatigue crack tip is shown in Fig. 7. To calculate the parameters presented in Fig. 7, the maximum of the temperature field near the crack tip was registered at each step of the experiment, and in equations (4)-(6) the origin of coordinates was displaced to a new position at each time moment. By comparing the time evolution of plastic work and dissipative energy, the deformation process corresponding to the transition from the stable state to the unstable state of crack propagation can be divided into three parts.

The first stage lasts for approximately 1.3 second; correlation between two curves is good. From 1.3 second to 5 seconds, the dissipation energy increases slowly compared to the plastic work. Over this period, the stored energy is monotonically accumulated in the deformed material and transformed into the potential energy of lattice distortion. At the last moments before fracture, the heat dissipation energy increases jumpwise and reaches the value of plastic work. At this time the rate of plastic work is less than the rate of heat dissipation energy. It can be assumed that the damage accumulation mechanism is changed, and the material approaches the fracture stage, where the role of macroscopic displacements is essential, and the energy dissipation increases significantly. We can suppose that the plastic work at this moment cannot be described by traditional HRR-solution (4), because this model does not agree with the effect of a jumpwise increase in the dissipation energy. So, the stored energy calculated at this moment should be corrected.

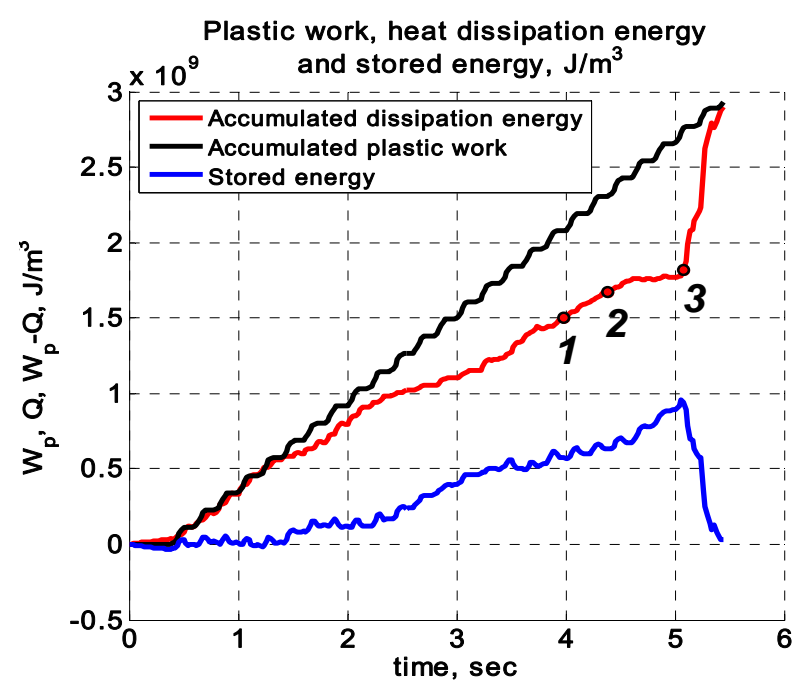

Figure 7: Time dependence of plastic work, heat dissipation energy and stored energy at the point near the crack tip (1, 2, 3 - time points for $\beta$-distribution in Fig. 8).

The data obtained for the examined material indicate that the stored energy reaches a critical value, after which the HRR solution is unable to describe the behavior of plastic work near the crack tip. We suppose that the stored energy observed at the last moment before fracture is equal to some constant that indicates the approaching material destruction. 


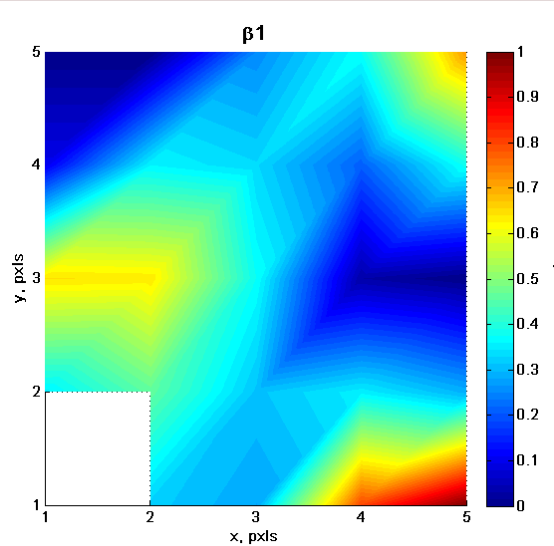

1

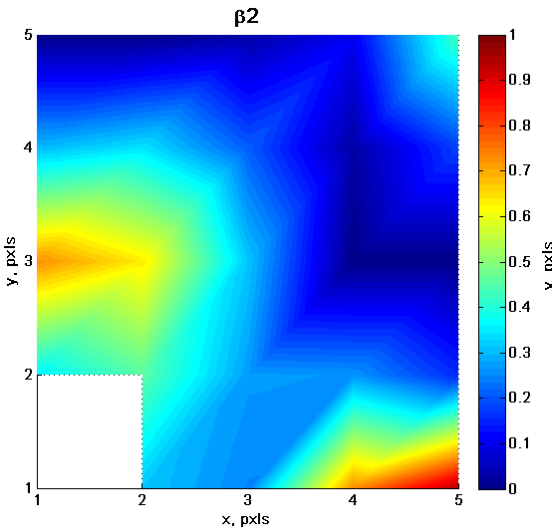

2

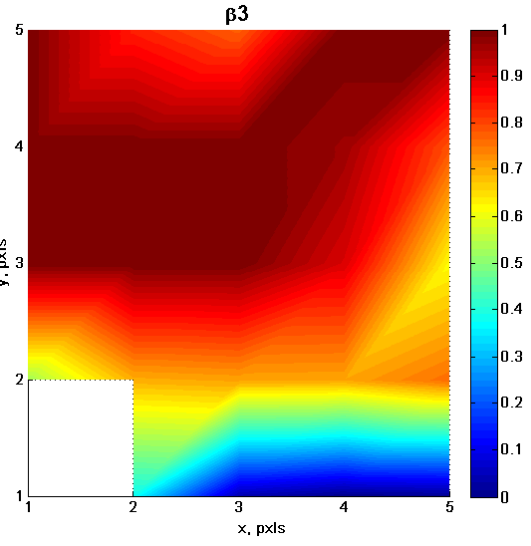

Figure 8: The first coordinate quarter of the space $\beta$-distribution at different time moments is shown in Fig. 7. The crack propagates along the $x$-axis.

We introduce the parameter $\beta$ to evaluate the value of stored energy in metals:

$$
\beta(x, y, t)=\frac{W_{p}(x, y, t)-Q(x, y, t)}{W_{p}(x, y, t)}
$$

The space distribution of the parameter $\beta$ at different time points is given in Fig. 8. A white pixel is the point of the crack tip where stresses are calculated based on the HRR-solution. Therefore, the plastic work has singularity at the crack tip. Pictures 1, 2 and 3 correspond to the points shown in Fig.7. It is seen that the dissipation energy increases depending on the plastic work, and the images of the space $\beta$-distribution are similar (pictures 1,2). Picture 3 indicates that the $\beta$ distribution changes, that is, in front of the crack tip an area with zero $\beta$ appears in the direction of crack propagation. Thus, the crack growth can be expected in the area of zero $\beta$.

The space distribution of the parameter $\beta$ makes it possible to access the direction of crack motion and to predict its growth up to the specimen failure. This circumstance allows us to use the parameter $\beta$ as a failure criterion that is based on the thermodynamic laws.

\section{CONCLUSION}

he infrared technique has been applied to investigate the effect of heat dissipation under quasi-static loading and its localization at the crack tip under cyclic loading. The original data processing algorithm developed earlier has allowed us to calculate the values of heat dissipation at the crack tip and to determine the area of plastic deformation localization on the surface of a smooth sample during the tensile test. A method has been developed for determining the stored energy and the parameter $\beta$, which could be used as a thermodynamic fracture criterion. The spatial and time distribution of the stored energy has been calculated using the infrared data. The damage parameter is taken as the ratio between the stored energy and the work of plastic deformation. For both the weakened and smooth samples, it has been found that the stored energy is accumulated in titanium specimens undergoing fatigue tests, and the value of stored energy is equal to zero when failure concentration reaches the critical value corresponding to specimen fracture. The obtained results yield information that can be used to develop engineering methods for analyzing the actual state of structures that undergo real loading.

\section{ACKNOWLEDGEMENTS}

1

his work was supported by the grant of the President of Russian Federation for support of young Russian scientists and leading scientific schools (MD-2684.2012.1) and RFBR (grant № 11-01-96005). 


\section{REFERENCES}

[1] V. E. Panin, Yu.V. Grinyaev, V. V. Danilov et al, Structural levels of plastic deformation and fracture. Novosibirsk: Nauka, (1990) 255 (in Russian).

[2] V. V. Rybin, Severe plastic deformation and failure of metals. M.:Metalurgiya, (1986) 224 (in Russian).

[3] V. S. Ivanova, V. F. Terentiev, The nature of the fatigue of metals. Moscow «Metallurgy», (1975) 456 (In Russian)

[4] V.T. Troshchenko, Deformation and fracture of metals under high cyclic loading. Kiev: «Naukova Dumka», (1981) 344 (In Russian)

[5] V.V. Fedorov, Thermodynamic aspects of strength and fracture of solids. Tashkent: «FAN» Uz SSR, (1979) 168 (In Russian)

[6] G. Curti, G. La Rosa, M. Orlando, A. Risitano, In: 14th AIAS Italian National Conference, Catania, Italy, (1986) 211 (in Italian).

[7] G. La Rosa, A. Risitano, Int. Journal of F., 22 (2000) 65.

[8] M. P. Luong, Nuclear Engineering and Design, 158 (1995) 363.

[9] M. B. Bever, D. L. Holt, A. L. Tichener, Prog. Mat. Sci., 17 (1973) 1.

[10] W. Oliferuk, A. Korbel, W. Bochniak, Materials Science and Engineering A, 319-321 (2001) 250.

[11] O. Plekhov, N. Saintier, T. Palin-Luc, S. Uvarov, A. Naimark, Material Science and Engineering A, $462(1)(2007) 367$.

[12] M. Bannikov, A. Terekhina, O. Plekhov, Vestnik Permskogo gosudarstvennogo tehnicheskogo universiteta. Mehanika, 2 (2011) 14. (In Russian).

[13] A.Yu. Fedorova, M.V. Bannikov, O.A. Plekhov, Fracture and Structural Integrity, 21 (2012) 46.

[14] J. Hodowany, G. Ravichandran, A.J. Rosakis, P.Rosakis, Exp Mech, 40(2) (2000) 113.

[15] O. Plekhov, S. Uvarov, O. Naimark, Strength of materials, 1(391) (2008) 101.

[16] Yu.G. Matvienko, V.G. Avramenko, Deformation and fracture of materials, 10 (2009) 2. (In Russian) 